\title{
Simulations of dense-snow avalanches on deflecting dams
}

\author{
Fridtjov Irgens, ${ }^{1}$ Bonsak Schieldrop, ${ }^{2}$ Carl B. Harbitz, ${ }^{3}$ Ulrik Domaas, ${ }^{3}$ Runar Opsahl ${ }^{3}$ \\ ${ }^{1}$ Department of Applied Mechanics, Thermo- and Fluid Dynamics, Norwegian University of Science and Technology, \\ $\mathcal{N}-7034$ Trondheim, Norway \\ ${ }^{2}$ Industrial Hydro- and Aero Dynamics, Herman Fossgt. 29, N-0171 Oslo, Norway \\ ${ }^{3}$ Norwegian Geotechnical Institute, P.O.Box 3930 Ullevaal Hageby, N-0806 Oslo, Norway
}

\begin{abstract}
Two models simulating snow avalanches impacting retaining dams at oblique angles of incidence are presented.

First, a lumped-mass model applying the Voellmy-Perla equation is used to calculate the path of the centre-of-mass along the side of a retaining dam.

Secondly, a one-dimensional continuum model, applying depth-integrated equations of balance of mass and linear momentum, is expanded to take into account that real avalanche flows are three-dimensional. The centre-line of the avalanche path is determined by the flowing material as it progresses down the channelized avalanche path. The nonlinear constitutive equations comprise viscosity, visco-elasticity and plasticity.

Both models are calibrated by simulations of a registered avalanche following a strongly curved channel. The path and the run-up height of the avalanche on the natural deflecting dam with oblique angle of incidence as calculated by the two models, are compared with the observations made.
\end{abstract}

\section{LIST OF SYMBOLS}

\section{Centre-of-mass model}

$g_{\mathrm{x}}, g_{\mathrm{y}}, g_{\mathrm{z}}, g$ Gravitational force per unit mass

$M / D \quad$ Mass-to-drag ratio

$R \quad$ Radius of curvature of the centre-of-mass path line along the wall

$r$

$r \quad$ Vertical run-up height above the base line of the deflecting dam, measured in a vertical cross section perpendicular to the horizontal projection of the base line of the deflecting dam

\section{Velocity of centre-of-mass}

Centre-of-mass velocity immediately before impact

\section{One-dimensional continuum model for three-di- mensional avalanche flow}

\begin{tabular}{|c|c|}
\hline$b_{\mathrm{xy}}$ & $\begin{array}{l}\text { Centrifugal force per unit mass in vertical tan- } \\
\text { gent plane }\end{array}$ \\
\hline$g$ & Gravitational force per unit mass \\
\hline$g_{\mathrm{e}}$ & Effective gravitational force per unit mass \\
\hline$h$ & Flow height \\
\hline$m$ & Shear viscosity \\
\hline$m_{1}, m_{2}$ & Visco-elasticities \\
\hline$n$ & Material power law exponent \\
\hline$P_{\mathrm{i}}$ & Station points on the centre line \\
\hline$p_{\mathrm{e}}$ & Effective pressure \\
\hline$p_{\mathrm{u}}$ & Pore pressure \\
\hline$Q_{\mathrm{i}}$ & $\begin{array}{l}\text { Path points defining the centre line of the ava- } \\
\text { lanche terrain }\end{array}$ \\
\hline$R$ & Radius of computational cross sectional profile \\
\hline$\nu_{\mathrm{a}}$ & Average velocity through flow cross section \\
\hline$\nu_{\mathrm{x}}, \nu_{\mathrm{y}}, \nu_{1}$ & Velocity components \\
\hline$w$ & Computational flow width \\
\hline$X Y Z, x y z$ & Cartesian coordinates \\
\hline$\alpha_{\mathrm{X}}$ & Slope of horizontal projection of centre line \\
\hline$\theta$ & $\begin{array}{l}\text { Angle of inclination of the free surface of the } \\
\text { avalanche }\end{array}$ \\
\hline$\kappa_{\mathrm{XZ}}$ & Curvature in t he horizontal plane \\
\hline$\kappa_{\mathrm{xy}}$ & Curvature in the vertical tangent plane \\
\hline$\mu$ & Dry-friction coefficient \\
\hline$\rho$ & Mass density \\
\hline$\sigma, \tau$ & Normal stress and shear stress \\
\hline$\phi$ & $\begin{array}{l}\text { Slope of centre line in the vertical tangent } \\
\text { plane }\end{array}$ \\
\hline
\end{tabular}

\section{INTRODUCTION}

Increased human activity in mountain regions, deforestation from pollution, forestry and ski resorts, as well as a reduced acceptance of living in regions exposed to snow 
avalanches have caused a growing need for protection against avalanches. Such protection is more and more often obtained by constructing retaining dams to influence the dense-snow avalanche course. Better knowledge and understanding of terrain deflection of dense-snow avalanches will improve the design of deflecting dams.

The first attempt to formulate a general theory of densesnow avalanche motion was made by Voellmy (1955) and this theory is still widely used. Both statistical and comparative models for run-out distance computations, as well as dynamic models for avalanche motion simulations, are now developed. However, no universal model has so far been made. The dynamics of avalanches are complex, involving both fluid, particle and soil mechanics. The limited amount of data available from real events makes it difficult to evaluate or calibrate existing models. Often several models with different physical descriptions of avalanche movement can all fulfil the deficient observational records.

In spite of the uncertainties with which the existing models are encumbered, simulations of avalanches impacting retaining dams at oblique angles of incidence have to take into account that real avalanche flows are three-dimensional. Neither the run-up heights pioneered by Voellmy (1955) nor the leading-edge models by Hungr and McClung (1987) or Takahashi and Yoshida (1979) (Takahashi, 1991) contain deflection effects. This is also the case for the paper by Chu and others (1995) on experiments on granular flows to predict avalanche run-up. Nohguchi (1989) has developed a three-dimensional dense-snow avalanche centre-of-mass model based on the equations of Voellmy (1955), while Sassa (1988) has developed a geotechnical quasi-three-dimensional continuum model. Lang and Leo (1994) developed a quasi-threedimensional dense-snow avalanche model. However, according to the originators it is still unknown whether the model can represent naturally occurring events.

Effect of natural deflecting dams on reported avalanches are described by Harbitz and Domaas (1997). The observations indicate that the height difference between the gully floor and the upper limit of extension on deflecting terrain formations might exceed $60 \mathrm{~m}$ for large avalanches (estimated volume $>100000 \mathrm{~m}^{3}$ ) reaching a velocity of more than $40 \mathrm{~m} \mathrm{~s}^{-1}$ in the avalanche track.

In this paper, we present two models of snow avalanches impacting on retaining dams at oblique angles of incidence. The first is a centre-of-mass model based on a Voellmy type of resistance force. In the second model, we extend the simulation model of Norem and others (1989), for one-dimensional avalanche flow parallel to a vertical plane, to avalanche flows following a three-dimensional channel with varying width. The material model used was first presented by Norem and others (1987) and a discussion on the importance of the physical parameters has been given by Irgens and Norem (1996).

\section{GENTRE-OF-MASS MODEL FOR AVALANGHE MOTION ALONG THE SIDE OF A RETAINING DAM}

A centre-of-mass model for avalanche motion along the side of a retaining dam was developed by B. Schieldrop (personal communication, 1996) in co-operation with the Norwegian Geotechnical Institute. Strictly speaking the centre-of-mass is representative of the frontal part of the https://doi.org/10.3189/1998AoG26-1-265-271 Published online by Cambridge University Press slide, projected onto the terrain (the total avalanche centre-of-mass may not even reach the dam). As in the model of Nohguchi (1989) for centre-of-mass motion on a three-dimensional surface of arbitrary configuration, the equations are derived from classical mechanics, including a resistance force represented by a dynamic drag and a Coulomb friction (as in the Voellmy (1955) model). However, a lumped-mass consideration does not comprise any dynamic effects of the avalanche extension. Hence, the model results will anyhow be encumbered with obvious restrictions. For these reasons, it was preferable to perform a simplified-geometry study of the influence of avalanche-impact velocity, terrain inclination, dam configuration, and dam orientation on avalanche-course deflection and run-up height along a deflection dam. An additional advantage of a simplified-geometry study is that the deflecting dam does not have to be superposed on a complex digital terrain.

The simplified geometry consists of a plane terrain of inclination $b$ and the upper plane wall of the deflecting dam, oriented by its angle relative to the terrain, $y$, and the angle between the base line of the wall (the $x$ axis) and the terrain contour lines, $j$, Figure 1.

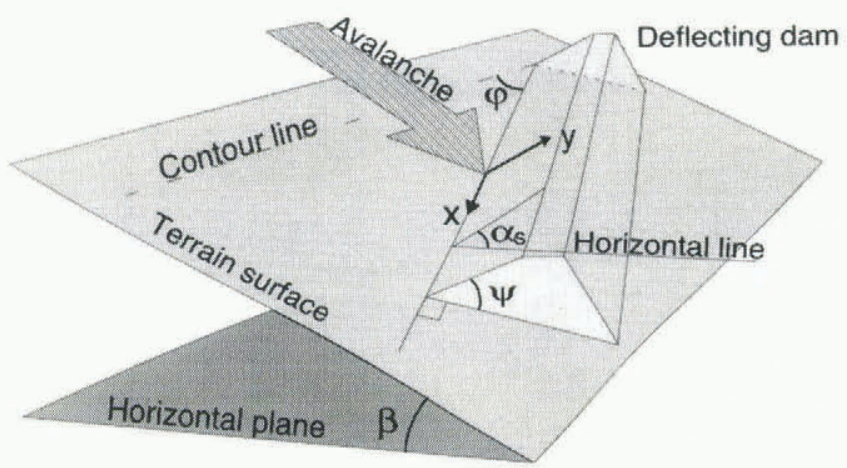

Fig. 1. Simplified geometry configuration for centre-of-mass model.

The tangential and normal components of the centre-ofmass momentum equations are

$$
\frac{d v}{d t}=g_{\mathrm{x}} \cos \gamma-g_{\mathrm{y}} \sin \gamma-{ }_{\mathrm{z}}-D / M \nu^{2}
$$

and

$$
\frac{\nu^{2}}{R}=g_{\mathrm{x}} \sin \gamma-g_{\mathrm{y}} \cos \gamma
$$

respectively, where $\nu$ is the centre-of-mass velocity at time $t$, $g_{\mathrm{x}}=g \sin \beta \sin \varphi, g_{\mathrm{y}}=-g(\cos \beta \sin \psi-\sin \beta \cos \varphi \cos \psi)$, and $g_{\mathrm{z}}=-g(\cos \beta \cos \psi+\sin \beta \cos \varphi \sin \psi)$ are components of the gravitational force per unit mass, $g$, in the upper wall plane, $g_{\mathrm{x}}$ along and $g_{\mathrm{y}}$ normal to the base line respectively, while $g_{\mathrm{z}}$ is the component normal to the wall plane. $\gamma$ is the angle between the centre-of-mass path tangent line and the base line, $\mu$ is the dry-friction coefficient, $M / D$ is the massto-drag ratio described by Perla and others (1980) and $R$ is the radius of curvature of the centre-of-mass path line on the wall. By means of the kinematic condition $\nu^{2} / R=-\nu(d / d t)$ and the transcription $(d v / d t=(d \nu / d \gamma)(d \gamma / d t)$, Equations (1) and (2) can be combined into:

$$
\frac{d \nu}{d \gamma}=-\frac{\left(g_{\mathrm{x}} \cos \gamma-g_{\mathrm{y}} \sin \gamma-\mu g_{\mathrm{z}}-D / M \nu^{2}\right) \nu}{\left(g_{\mathrm{x}} \sin \gamma+g_{\mathrm{y}} \cos \gamma\right)},
$$

which is solved numerically by a fourth order Runge-Kutta procedure. The angle $\gamma$ is reduced by constant increments $d \gamma$ 
throughout the simulations. For each new pair of $(\nu, \gamma)$ values, the centre-of-mass is moved a distance $d s=\nu d t$ along the upper wall in the direction determined by the value of $\gamma$. The time increment $d t=-\nu d \gamma /\left(g_{\mathrm{x}} \sin \gamma+g_{\mathrm{y}} \cos \gamma\right)$ is found by combining Equation (2) and the kinematic condition above.

Also the effects of energy loss due to impact may be investigated. Without any loss, initial values are $\gamma_{0}=\pi / 2-\varphi$ and $\nu_{0}=\nu_{\mathrm{T}}$, where $\nu_{\mathrm{T}}$ is the centre-of-mass velocity immediately before impact. If the centre-of-mass velocity component normal to the upper wall is lost during the impact, initial values are $\gamma_{0}=\tan ^{-1}(\cos \psi / \tan \varphi)$ and $\nu_{0}=\nu_{\mathrm{T}}$ $\left(\sin ^{2} \varphi+\cos ^{2} \varphi \cos ^{2} \psi\right)^{\frac{1}{2}}$.

\section{ONE-DIMENSIONAL CONTINUUM MODEL FOR THREE-DIMENSIONAL AVALANCHE FLOW}

\subsection{Physical and mathematical model}

The avalanche channel path is approximated by a set of volume elements with varying widths, compensating for converging and diverging effects in a real avalanche flow. Furthermore, horizontal centrifugal effects due to the curvature of the horizontal projection of the path are taken into account. A preliminary version of the simulation procedure, in which the centre-line of the avalanche path had to be specified prior to the simulation, has been presented by Irgens (19th ICTAM, Japan, August 1996, to be published). The main feature of the model is the fact that the centre-line of the avalanche is a space curve, which is determined by the terrain and in the present improved version also by the dynamics of the flowing material. This is in contrast to the three-dimensional model of Sassa (1988) and Lang and Leo (1994), where the centre-line is a curve in a vertical plane. Tentative two-dimensional models for three-dimensional dense snow avalanche flow are being developed at the Swiss Federal Institute for Snow and Avalanche Research (personal communication, U. Gruber, 1997) and CEMAGREF, France (personal communication, M. Naaim, 1997).

A representation of the three-dimensional avalanche topography is shown in Figure 2. The geometry of an avalanche channel is defined by a preliminary centre-line space curve and terrain profiles in cross sections perpendicular to this line. The centre-line is specified by a selected number of path points, $Q_{1}, \ldots, Q_{\mathrm{n}}$, at the bottom of the profiles and defined by Cartesian coordinates $(X, Y, Z)$. The projections of the centre-line in the $X Y$ - and $X Z$ planes are replaced by cubic splines. The centre-line is then subdivided into a chosen number of subsegments by station points, $P_{1}, \ldots, P_{\mathrm{m}}$.

The cross-sectional terrain profile at each path point $Q_{\mathrm{i}}$ is approximated by a circle of radius $R_{\mathrm{i}}(X)$ as shown in Figure 3 . The radii $R_{1}, \ldots, R_{\mathrm{m}}$ of similar profiles at the station points, $P_{1}, \ldots, P_{\mathrm{m}}$ are found from a cubic spline through points with Cartesian coordinates $(X, R)$ for the path points, $Q_{1}, \ldots, Q_{\mathrm{n}}$. By this procedure the real avalanche channel is replaced by a set of elements between the crosssectional profiles. The avalanche of the flowing material is defined by a subset of these elements filled with snow. The height of the snow is given by $h_{\mathrm{i}}(X, t)$ at the station points $P_{\mathrm{i}}$. The circular terrain profile shown in Figure 3 represents the terrain profile both at the path points, $Q, \ldots, Q_{\mathrm{n}}$ and the station points, $P_{1}, \ldots, P_{\mathrm{m}}$.

The profile of the flowing material through the cross sec-

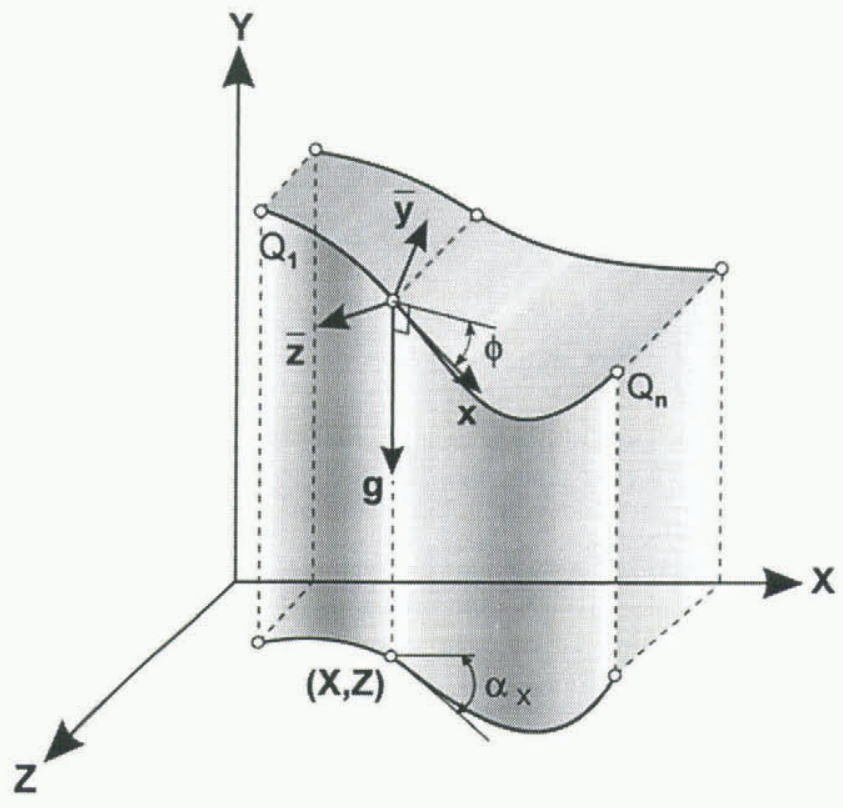

Fig. 2. Centre-line of three-dimensional avalanche. $Q_{1}, \ldots$, $Q_{\mathrm{n}}$ are the path points defining the centre-line. $X Y$ is a vertical plane, and $X Z$ is a horizontal plane. The $x$ axis is in the direction of the flow. The $x \bar{y}$ plane is vertical. $\phi$ is the angle of flow inclination with respect to the horizontal plane. $\alpha_{\mathrm{X}}$ is the slope of the projection of the avalanche centre-line in the $X Z$ plane with respect to the $X$ axis. $g$ is the gravitational force per unit mass.

tion is approximated by a circular segment. Due to centrifugal forces the trace of the free surface in the cross section will be inclined with respect to the horizontal plane. The angle of inclination $\theta$ defines the origin of the local coordinate systems $x y z$ and $x \overline{y z}$ as shown in Figure 3. The $x$ axis is tangent to the path curve, and the $x \bar{y}$ plane is vertical. The origins

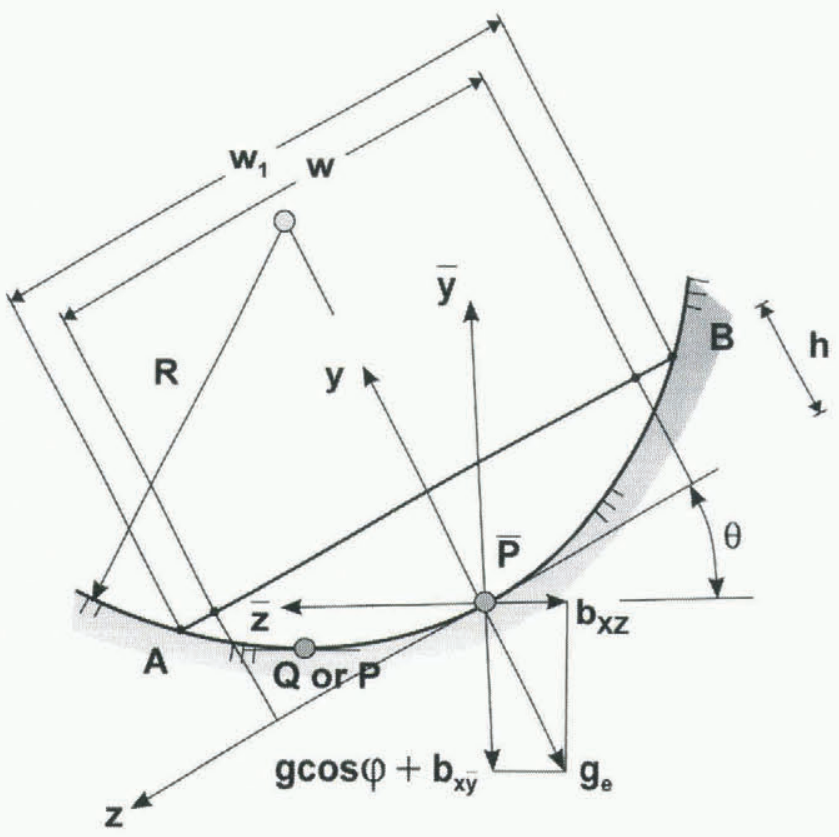

Fig. 3. Circular segment cross-sectional profile at a path point $Q$ or a station point $P . \bar{P}$ is the adjusted station point. $\theta$ is the angle of inclination of the profile, $b_{\mathrm{XZ}}$ is the centrifugal force per unit mass in the $X Z$ plane, $h$ is the height of the flow and is determined by the flow. $w$ is the computational width of the corresponding rectangular profile, and is determined by $h$ and the radius $R . w_{1}$ is the width of the circular segment. 
are taken to be adjusted station points $\bar{P}_{\text {in }}$ with new global coordinates $\left(X_{\mathrm{i}}, Y_{\mathrm{i}}, Z_{\mathrm{i}}\right)$ for the calculated centre-line of the avalanche. The circular-segment cross section of the flowing material is further replaced by a rectangular cross section of height $h$ and computational width $w$, and with the same cross-sectional area $A$ as the circular cross section. The assumption of a circular-segment profile implies an interdependence between the flow width $w_{1}(X, t)$ and the flow height $h(X, t)$. The slope $\alpha_{\mathrm{X}}$ and the curvature $\kappa_{\mathrm{XZ}}$ in the horizontal $X Z$ plane, and the slope $\phi$ and the curvature $\kappa_{\mathrm{x} \bar{y}}$ in the vertical $x \bar{y}$ plane are all computed from the coordinates of the stations $P_{\mathrm{i}}$ and based on central-difference formulas.

The projected curved motion of the flowing material in the $X Z$ plane is responsible for a horizontal centrifugal force component $b_{\mathrm{XZ}}$ per unit mass.

$$
b_{\mathrm{XZ}}=\left(\nu_{\mathrm{a}} \cos \phi\right)^{2} \kappa_{\mathrm{XZ}}
$$

where $\nu_{\mathrm{a}}$ is the average velocity through the cross section of the flow. Due to this centrifugal force the free surface of the flowing material will be inclined with respect to the horizontal $\bar{z}$ axis at each station profile. The gravitational force $g$ per unit mass has a driving component $g \sin \phi$ in the $x$ direction and a component $g \cos \phi$ in the $\bar{y}$ direction. To the latter component we add a centrifugal-force component in a vertical plane

$$
b_{\mathrm{x} \overline{\mathrm{y}}}=\left(\nu_{\mathrm{a}} \cos \phi\right)^{2} \kappa_{\mathrm{x} \overline{\mathrm{y}}}
$$

The effective gravitational force, $g_{\mathrm{e}}$, in the $\bar{y} \bar{z}$ plane is the resultant of these forces

$$
g_{\mathrm{e}}=\sqrt{\left(g \cos \phi+b_{\mathrm{x} \bar{y}}\right)^{2}+b_{\mathrm{XZ}}^{2}}
$$

This body force defines the angle of inclination $\theta$ of the free surface of the avalanche, which is determined from

$$
\theta=\arctan \left(\frac{b_{\mathrm{xz}}}{g \cos \phi+b_{\mathrm{x} \bar{y}}}\right)
$$

This angle determines the directions of the coordinate axes $y$ and $z$ in the $\bar{y} \bar{z}$ plane. The flow is now considered to be two-dimensional with the velocity field given by the two components $\nu_{\mathrm{x}}(X, y, t)$ and $\nu_{\mathrm{y}}(X, y, t)$.

For the sake of simplicity, the complete version of the simulation model presents two special options as alternatives: (1) For highly cohesive material extensional flow with a uniform streamwise velocity $\nu_{\mathrm{x}}=v_{\mathrm{o}}(X, t)$ is assumed. The constitutive equations contain terms representing active- and passive-pressure contribution. (2) When cohesion may be neglected, shear flow and the no-slip condition $\nu_{\mathrm{x}}=0$ on the bed surface $y=0$ are assumed. On the free surface, $y=h(X, t)$, the normal stress must be equal to the atmospheric pressure, which is assumed to be equal to the pore pressure $p_{\mathrm{u}}$, and the shear stress must be zero. The constitutive equations do not produce active- and passive-pressure terms in this case.

\subsection{Constitutive model}

A general discussion of the constitutive model may be found in Norem and others (1987, 1989). For a two-dimensional steady gravity-driven shear flow the equation of motion in the streamwise direction yields the velocity field

$$
\nu_{\mathrm{x}}(y)=\nu_{0}+\nu_{1}\left[1-\left(1-\frac{y}{h}\right)^{\frac{1+n}{n}}\right]
$$

where $\nu_{0}$ is the velocity at the bed surface, $\nu_{1}$ is the velocity https://doi.org/10.3189/1998AoG26-1-265-271 Published online by Cambridge University Press of the free surface and $n$ is a material power-law exponent. The relevant shear and normal stresses for this flow in the $x$ and $y$ directions are

$$
\begin{gathered}
\tau_{\mathrm{xy}}=\left(a+\mu p_{\mathrm{e}}\right)+\rho m\left(\frac{\partial \nu_{\mathrm{x}}}{\partial y}\right)^{n} \\
\sigma_{\mathrm{x}}=-\left(p_{\mathrm{u}}+p_{\mathrm{e}}\right)+\rho\left(m_{1}-m_{2}\right)\left(\frac{\partial \nu_{\mathrm{x}}}{\partial y}\right)^{n} \\
\sigma_{\mathrm{y}}=-\left(p_{\mathrm{u}}+p_{\mathrm{e}}\right)-\rho m_{2}\left(\frac{\partial \nu_{\mathrm{x}}}{\partial y}\right)^{n}
\end{gathered}
$$

The material properties of the model are specified by the cohesion parameter $a$, the coefficient of dry friction $\mu$, the shear viscosity $m$, the exponent $n$, and the two visco-elasticities $m_{1}$ and $m_{2}$, representing the effect of normal stress differences. $p_{\mathrm{e}}$ is the effective pressure and $\rho$ is the density of the snow.

\subsection{Numerical simulation}

It is assumed that the equation of motion in the $y$ direction may be approximated by an equilibrium equation, which may be integrated to

$$
\sigma_{\mathrm{y}}=-p_{\mathrm{u}}-\rho g_{\mathrm{e}}(h-y)
$$

An expression for the effective pressure $p_{\mathrm{e}}$ is obtained by a comparison of Equations (11) and (10). The velocity in the streamwise direction $\nu_{\mathrm{x}}(X, y, t)$ is assumed to be approximately given by the steady-state function in Equation (8), where the velocity of the free surface $\nu_{1}$ is now assumed to be a function of $X$ and $t$. The equation of motion in the streamwise direction and the continuity equation are integrated in the $y$ direction. The assumed velocity profile $\nu_{\mathrm{x}}(X, y, t)$ is substituted into the integral equations. From previous two-dimensional simulations, it is known that with reasonable parameter values, the results are more or less similar for case 1 and case 2, referred to in section 3.1. For case 2, which is more easily implemented in the numerical model, the following differential equations are obtained

$$
\begin{aligned}
& \frac{\partial \nu_{\mathrm{a}}}{\partial t}=\frac{1}{h}\left\{h g \sin \phi-h \mu g_{\mathrm{e}}-\left(m-b m_{2}\right)\left[\nu_{\mathrm{a}} \frac{(1+2 n)}{n h}\right]^{n}\right. \\
&+ \frac{\nu_{\mathrm{a}}}{w} \frac{\partial\left(w h \nu_{\mathrm{a}}\right)}{\partial x}-\frac{1}{w} \frac{\partial}{\partial x}\left[\frac{2 w h \nu_{\mathrm{a}}^{2}(1+2 n)}{2+3 n}\right] \\
&\left.+\frac{\partial}{\partial x}\left[-g_{\mathrm{e}} \frac{h^{2}}{2}+m_{1}\left(\frac{\nu_{\mathrm{a}}(1+2 n)}{n h}\right)^{n} \frac{h}{2}\right]\right\} \\
& \frac{\partial h}{\partial t}=-\frac{1}{w+h F_{1}} \frac{\partial\left(w h \nu_{\mathrm{a}}\right)}{\partial x}
\end{aligned}
$$

where

$$
\begin{aligned}
F_{1} & =\left(\frac{R}{h}\right)^{2}\left[\left(1+\frac{h}{R}\right) \sqrt{2 \frac{h}{R}-\left(\frac{h}{R}\right)^{2}}\right. \\
& \left.-\arcsin \sqrt{2 \frac{h}{R}-\left(\frac{h}{R}\right)^{2}}\right]
\end{aligned}
$$

and

$$
\nu_{\mathrm{a}}=\frac{(1+n) \nu_{1}}{1+2 n} .
$$

To determine the motion, the equation of motion (12) together with the continuity equation (13) and the geometri- 


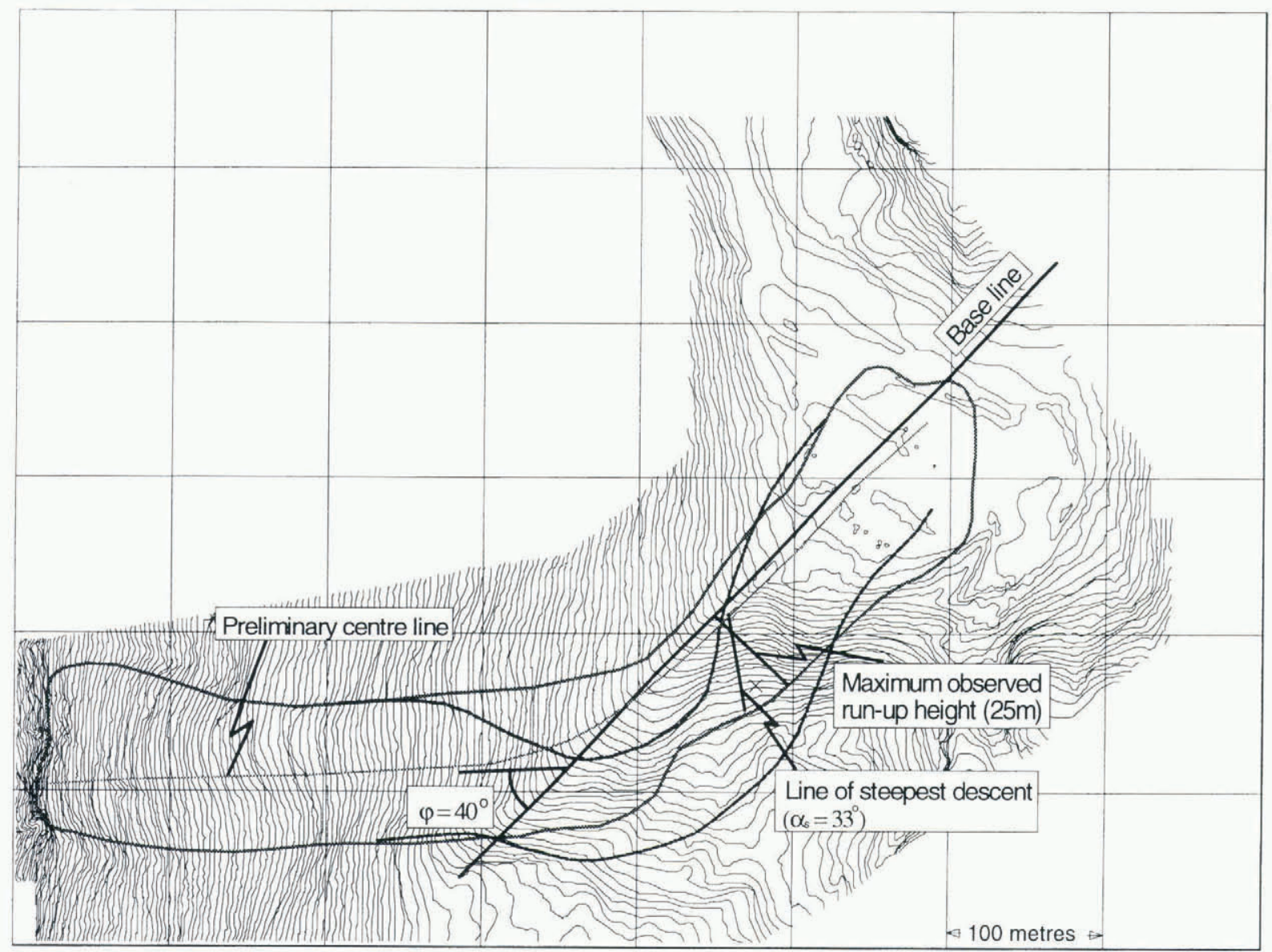

Fig. 4. Simulations of the 1986 Vassdalen avalanche by three-dimensional continuum model (dotted line) compared with the observations made (solid line). All angles and lines are horizontal projections. Location of observed maximum run-up height, measured in a vertical cross section perpendicular to the horizontal projection of the base line of the natural deflecting dam, indicated. Contour-line interval is $2 \mathrm{~m}$.

cal relation between the flow height and the flow width provide three equations for the three unknowns $\nu_{\mathrm{a}}, h$, and $w$. The partial differential equations are solved by a finite difference scheme with spatial central differences in the streamwise direction, and by a fourth order Runge-Kutta procedure with respect to time. The finite-difference scheme is Eulerian, requiring the following special procedure to make the avalanche progress along the path: The volume of snow passing through the front section at station $\bar{P}_{\mathrm{f}}$ fills the downstream subsegment, where the subscript $f$ denotes the number of the contemporary front station. When the accumulated volume exceeds the current value of the volume $h_{\mathrm{f}} \cdot w_{\mathrm{f}} \cdot \Delta x_{\mathrm{f}+1}$, where $\Delta x_{\mathrm{f}+1}$ is the distance between the stations $\bar{P}_{\mathrm{f}}$ and $\bar{P}_{\mathrm{f}+1}$, it is assumed that the avalanche front has advanced one subsegment. A similar procedure is applied to the tail of the avalanche.

Initially the snow is assumed to fill a certain number of volume elements and is at rest. The origins of the local coordinate systems $x y z$ are located at the station points $P$. As the motion starts and new volume elements are filled with snow, the origin $\bar{P}$ of the next front station is determined by the angle $\theta$ from Equation (7). The result is an avalanche that finds its path according to the terrain and the dynamics of the flow. The width $w_{1}$ of the circular segments in Figure 3 are found from a geometrical formula relating $h, R$, and $w_{1}$. The computer program is developed for personal computers.

\section{THE 1986 VASSDALEN AVALANCHE}

\subsection{The observed avalanche}

Extreme snow fall combined with strong winds and a cold period during the first part of the winter followed by temperature variations were the main triggers for the dry-snow avalanche in Vassdalen, Narvik, northern Norway on 5 March 1986. 16 soldiers were killed in the avalanche, which was therefore reported extensively (Lied, 1988). The mapping of the avalanche was accomplished shortly after the event, and was based mainly on location of snow deposits and injuries on the birch forest. The deposited snow masses, the severe deflection of the avalanche course, the eyewitnesses accounts, and the limited extension of the injuries all indicate that this was a dense dry-snow avalanche with an insignificant powder-snow cloud. For these reasons, the 1986 Vassdalen avalanche serves as a well-defined full-scale experiment regarding avalanche travel path and extension.

The fracture line of the avalanche was located $475 \mathrm{~m}$ a.s.l. The approximately $100 \mathrm{~m}$ wide release zone has an upward concave transversal terrain profile and an average inclination of $35.5^{\circ}$ above $375 \mathrm{~m}$ a.s.l. (Fig. 4). The terrain inclination along the base of the natural deflecting dam is approximately $\beta=26.5^{\circ}$. The angle between the base line of the wall and the terrain contour lines is estimated to $\varphi=50^{\circ}$. In the region where the avalanche obtains its max- 
imum run-up height, the line of steepest descent along the upper wall of the dam has an inclination of $\alpha_{\mathrm{s}}=33^{\circ}$. The deflecting terrain in the lower half of the track caused an absolute maximum run-up height above the base line of the wall of about $25 \mathrm{~m}$ measured in a vertical cross section perpendicular to the horizontal projection of the base line of the natural deflecting dam.

\subsection{The simulated avalanche}

\subsubsection{Simulations by centre-of-mass model}

Input values $\beta=26.5^{\circ}, \varphi=50^{\circ}, \alpha_{\mathrm{s}}=33^{\circ}$ and $\nu_{\mathrm{T}}=$ $28 \mathrm{~m} \mathrm{~s}^{-1}$ (where the velocity is deduced from the three-dimensional simulations described below), give $\psi=44.6^{\circ}$. Hence the simulation of the centre-of-mass motion along the wall including energy loss due to impact starts with deduced values $\nu_{0}=25.0 \mathrm{~m} \mathrm{~s}^{-1}$ and $\gamma_{0}=30.9^{\circ}$. With $\mu=0.2$ and $M / D=500 \mathrm{~m}$ (chosen clearly within the documented possible range of these values), the maximum calculated run-up height of the centre-of-mass above the base line of the deflecting dam, measured along the terrain in a cross section perpendicular to the base line of the natural deflecting dam, is $y_{\max }=16.0 \mathrm{~m}$ (Fig. 5). This corresponds to a maximum vertical run-up height above the base line of the deflecting dam, measured in a vertical cross section perpendicular to the horizontal projection of the base line of the natural deflecting dam, of $r_{\max }=7.7 \mathrm{~m}$, where

$$
r=y(\sin \psi \cos \beta-\cos \psi \sin \beta \cos \varphi+\tan \xi \sin \varphi \sin \beta)
$$

and

$$
\xi=\cos ^{-1}\left[\left(g_{\mathrm{y}}^{2}+g_{\mathrm{z}}^{2}\right)\left(g^{4}-g_{\mathrm{x}}^{2} g^{2}-g_{\mathrm{x}}^{2} g_{\mathrm{z}}^{2}\right)^{-\frac{1}{2}}\right] .
$$

This is the run-up height estimated from maps and intuitively pointed out in the terrain, and also the most convenient height in dam design.

Without energy loss due to impact, $\nu_{0}=28 \mathrm{~m} \mathrm{~s}^{-1}$ and $\gamma_{0}=40.0^{\circ}$. The corresponding maximum run-up height is now $y_{\max }=29.5 \mathrm{~m}$ or $r_{\max }=14.1 \mathrm{~m}$.

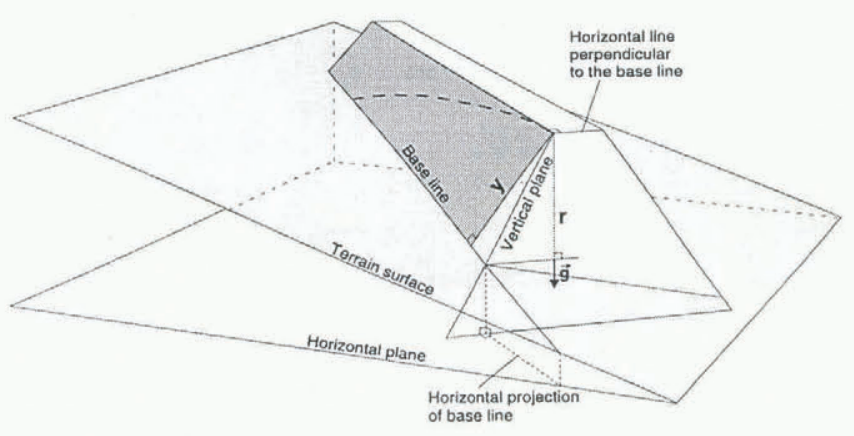

Fig. 5. Principal sketch of run-up heights on deflecting dam. Curved dotted line indicates outer extension of avalanche flow on the wall of the dam. $y$ is the run-up height measured along the terrain in a cross section perpendicular to the base line of the dam, while $r$ is the vertical run-up height measured in a vertical cross section perpendicular to the horizontal projection of the base line of the deflecting dam.

\subsubsection{Simulations by three-dimensional continuum model}

The input values to this model were chosen to give reasonable agreement between the simulated avalanche and the recorded path and the run-out distance of the real avalanche. The material parameters are: $\rho=300 \mathrm{~kg} \mathrm{~m}^{-3}, \mu=0.4$, $m=0.00146 \mathrm{~m}^{2}, m_{1}=0.0144 \mathrm{~m}^{2}, m_{2}=0.00144 \mathrm{~m}^{2}$ and https://doi.org/10.3189/1998AoG26-1-265-271 Published online by Cambridge University Press $n=2.0$. Ten path points were chosen to describe the centre-line of the preliminary path. The radii $R$ of the computational cross sections near the initiation of the avalanche and the run-out zone had to be estimated on the basis of the recorded width of the real avalanche. The fracture width and the curvature of the uppermost computational cross section implicitly define the fracture-height area.

\section{GALIBRATION AND GOMPARISON OF MODEL RESULTS}

For the centre-of-mass model a natural first assumption is that the centre-of-mass should reach half the run-up height observed in the terrain. This assumption is valid when the whole avalanche is climbing the wall of the deflecting dam with the masses equally distributed on both sides of the centre-line. Relatively good agreement with these conditions was one of the reasons why the Vassdalen avalanche was chosen for back calculations.

As described in section 4.2.1., the maximum run-up height calculated by the centre-of-mass model without energy loss due to impact is $14.1 \mathrm{~m}$, while the corresponding observed maximum run-up height was $25 \mathrm{~m}$ (Fig. 4), i.e. close agreement with the assumption above. Including energy loss due to impact reduces the calculated run-up height to $7.7 \mathrm{~m}$. Probably the correct maximum centre-ofmass run-up height is somewhere in between these extreme limits. The avalanche masses were probably distributed more towards the base of the deflecting terrain than indicated by a centre-line, hence reducing the upper value of $14.1 \mathrm{~m}$. On the other hand, due to snow compressibility and smooth terrain conditions, the lower extreme of $7.7 \mathrm{~m}$ based on total neglect of the centre-of-mass velocity component normal to the upper wall during impact, is probably too small.

Figure 4 shows the result of the simulation with the three-dimensional continuum model. In comparison with the recorded real avalanche the simulated avalanche is too sensitive to centrifugal effects. This may be due partly to the fact that a very crude approximation to the avalanche channel is used. The simulated avalanche channel in Figure 4 demonstrates the main features of the model: the centre-line is determined by the centrifugal effects, and the width of the avalanche is governed by the topography of the terrain. The maximum flow height is initially $3 \mathrm{~m}$, and increases to $4.5 \mathrm{~m}$ at the major bend, and becomes finally $3.5 \mathrm{~m}$ in the run-out zone.

\section{CONCLUSIONS}

The 1986 Vassdalen dense dry-snow avalanche is back calculated both by a centre-of-mass model applying the Voellmy-Perla equation, and a quasi-three-dimensional continuum model applying one-dimensional depth integrated equations of mass and linear momentum. The centre-of-mass path line calculated by the centre-of-mass model is in close agreement with the observations when energy loss due to impact is neglected. The model is applicable for studying the influence of terrain inclinations, impact velocity and dam configurations on the avalanche centre-of-mass path line. However, the model results are still encumbered with uncertainties, even though the parameters describing the features above are included. Whether 
the whole avalanche is climbing the wall depends on the same features. If a considerable part of the avalanche is not climbing the wall, the simulations should be made by consideration of a characteristic width of the slide representing the climbing fraction of the slide only. However, it is cumbersome to define an objective criteria for calculation of such a width. Besides the interaction with the masses not climbing the wall, being deflected or not, should also be taken into account. Hence, for future design of deflecting dams, there is hardly any alternative to (quasi) three-dimensional simulations.

The three-dimensional continuum model has in its present version several deficiencies. It may be difficult to model the terrain satisfactorily using the proposed avalanche channel. In future work a better approximation to the cross-sectional profiles will be attempted. From the present simulation it is clear that the model is too sensitive to centrifugal effects. The cubic-spline approximation of the centreline has unwanted consequences, as it sometimes introduce ripples in the centre-line which give rise to unrealistic curvatures and centrifugal effects.

\section{ACKNOWLEDGEMENTS}

Thanks are due to colleagues at the Norwegian Geotechnical Institute for their technical advice and discussions.

\section{REFERENCES}

Chu, T., G. Hill, D. M. McClung, R. Ngun and R. Sherkat, 1995. Experiments on granular flows to predict avalanche runup. Can. Geotech. . j.,
$32(2), 285-295$.

Harbitz, C. and U. Domaas. 1997. Runout distance - mapping of natural deflecting dams. Oslo, Norges Geotekniske Institutt. (NGI Rapport 581210-1.)

Hungr, O. and D. M. McClung. 1987. An equation for calculating snow avalanche run-up against barriers. International Association of Hydrological Sciences Publication 162 (Symposium at Davos 1986 - Avalanche Formation, Movement and Effects), 605-612.

Irgens, F. and H. Norem. 1996. A discussion of the physical parameters that control the flow of natural landslides. 7th International Symposium on Landstides, Trondheim, Norway. Proceedings. Vol. 2. Rotterdam, A. A. Balkema, 1251-1256.

Lang, R. M. and B. R. Leo. 1994. Model for avalanches in three spatial dimensions: comparion of theory to experiments. CRREL Rep. 94-5.

Lied, K. 1988. The avalanche accident at Vassdalen, Norway, 5 March 1986. Cold Reg. Sci. Technol., 15 2), 137-150.

Nohguchi, Y. 1989. Three-dimensional equations for mass centre motion of an avalanche of arbitrary configuration. Ann. Glaciol., 13, 215-217.

Norem, H., F. Irgens and B. Schieldrop. 1987. A continuum model for calculating snow avalanche velocities. International Association of Hydrological Sciences Publication 162 (Symposium at Davos 1986 - Avalanche Formation, Movement and Effects), 363-379.

Norem, H., F. Irgens and B. Schieldrop. 1989. Simulation of snow-avalanche flow in run-out zones. Ann. Glaciol., 13, 218-225.

Perla, R., T. T. Cheng and D. M. McClung. 1980. A two-parameter model of snow-avalanche motion. 7. Glaciol., 26 (94), 197-207.

Sassa, K. 1988. Geotechnical model for the motion of landslides. In Bonnard, C., ed. Landslides. Proceedings, 5th International Symposium on Landslides, 10-15 fuly 1988, Lausanne. Switzerland. Vol. 1. Rotterdam, A. A. Balkema, 1153-1158.

Takahashi, T. 1991. Debris flow. Rotterdam, A. A. Balkema. International Association for Hydraulic Research. (IAHR Monograph.)

Takahashi, T. and H. Yoshida. 1979. Study on the deposition of debris flows. Part l. Deposition due to abrupt change of bedslope.]. Japan, Kyoto University. Disaster Prevention Research Institute. (Annals 22 B-2.) In fapanese with English summary.7

Voellmy, A. 1955. Über die Zerstörungskraft von Lawinen. Schweiz. Bauztg., $73(12 / 15 / 17 / 19), 159-165,212-217,246-249,280-285$ 